\title{
A COMUNIDADE COMO LOCAL DE PROTAGONISMO NA INTEGRAÇÃO ENSINO-SERVIÇO E ATUAÇÃO MULTIPROFISSIONAL
}

\author{
THE COMMUNITY AS A PLACE OF LEADERSHIP IN EDUCATION-SERVICE INTEGRATION AND \\ MULTI-PROFESSIONAL PERFORMANCE
}

\section{LA COMUNIDAD COMO LOCAL DE PROTAGONISMO EN LA INTEGRACIÓN ENSEÑANZA-SERVICIOY ACTUACIÓN MULTIPROFESIONAL}

\author{
Anízia Aguiar Neta ${ }^{1}$ \\ Maria do Socorro Costa Feitosa Alves ${ }^{2}$
}

Resumo A discussão atual sobre educação e saúde tem mostrado a necessidade de maior integração entre os serviços de saúde e a academia. A Política Nacional de Educação Permanente em Saúde propõe a disseminação de capacidade pedagógica no Sistema Único de Saúde, de modo que a rede pública de saúde passe a constituir-se num espaço de ensino-aprendizagem no exercício do trabalho. O objetivo que se estabeleceu foi o de conhecer o processo de integração entre o ensino e o serviço de saúde na Atenção Básica em Saúde, a partir da atuação dos preceptores no processo de formação dos estudantes dos cursos de graduação da área da saúde na Universidade Federal do Rio Grande do Norte. A entrevista semiestruturada e a observação direta foram as técnicas escolhidas, e a análise dos dados, feita com base na abordagem hermenêutica-dialética, mostrou que a atuação dos preceptores se constitui em uma importante estratégia para viabilizar a integração ensino-serviço. A conclusão indica para a compreensão de que os profissionais envolvidos na preceptoria se educam enquanto educam, bem como de que o processo educativo é permeado por saberes e experiências heterogêneas, fator altamente favorável à formação dos estudantes e profissionais.

Palavras-chave educação; saúde; política nacional de educação permanente em saúde; preceptoria.
Abstract The current discussion on education and health has shown the need for greater integration between health services and the academy. The National Policy of Permanent Health Education proposed the dissemination of pedagogical capacity in the National Health System, so that the public health system would become a teaching and learning space while one performs his or her work. The goal was to get to know the process of integration between education and health services in Primary Health Care, from the actions of professors in the training process of students in the undergraduate courses in health care at the Federal University Rio Grande do Norte. Semi-structured interviews and direct observation were the techniques chosen, and data analysis, based on the hermeneutic-dialectic approach, showed that the professor's performance is an important strategy to facilitate integration between teaching and service. The conclusion points to the realization that the professionals involved in teaching are educated while they educate, and that the educational process is permeated by heterogeneous knowledge and experience, a highly favorable factor for the training of students and professionals. Keywords Education; health; national policy on continuing education in health; professorship. 


\section{Introdução}

Houve um tempo em que formar profissionais para o trabalho na área da saúde resumia-se a oferecer cursos pensados a partir da lógica da 'capacitação e atualização de recursos humanos'. A ideia era treinar habilidades para atender a um currículo estabelecido com base num conceito de saúde que se encontrava desarticulado das necessidades reais dos usuários e, consequentemente, das necessidades de formação dos profissionais. O momento atual, entretanto, permite uma nova compreensão sobre a educação da saúde, com ilimitadas possibilidades de inventar e disseminar tecnologias educacionais no setor (Ceccim, 2005).

A Política Nacional de Educação Permanente em Saúde (PNEPS) propõe, a partir de 2003, a disseminação de capacidade pedagógica no Sistema Único de Saúde (SUS), de modo que a rede pública de saúde passe a constituir-se num espaço de ensino-aprendizagem no exercício do trabalho, o que significa que a formação dos estudantes e profissionais deve acontecer dentro do próprio serviço, em situações reais (Ceccim, 2005).

A necessidade de uma política capaz de contemplar a complexidade do SUS levou o Ministério da Saúde a elaborar orientações e diretrizes que visam assegurar a educação dos trabalhadores, estabelecendo que "A Educação Permanente é aprendizagem no trabalho, onde o aprender e o ensinar se incorporam ao cotidiano das organizações e ao trabalho" (Brasil, 2006, p. 20).

Ao propor uma educação que se desenvolve no próprio trabalho, o Ministério da Saúde afirma que:

A definição de uma política de formação e desenvolvimento para o Sistema Único de Saúde, seja no âmbito nacional, estadual, regional e mesmo municipal, deve considerar o conceito de Educação Permanente em Saúde e articular as necessidades dos serviços de saúde, as possibilidades de desenvolvimento dos profissionais, a capacidade resolutiva dos serviços de saúde e a gestão social sobre as políticas públicas de saúde (Brasil, 2006, p. 20).

Estabeleceu-se, portanto, a exigência de uma nova postura para lidar com a questão da educação dos profissionais da saúde. O rompimento com a ideia de 'qualificar recursos humanos' na perspectiva de cursos e treinamentos para aperfeiçoar o trabalho representou um marco na história da educação dos profissionais da saúde. O foco das ações educativas passou a ser as necessidades de saúde da população, de maneira a garantir que os usuários do sistema público sejam amplamente contemplados. Além disso, a PNEPS relaciona a formação dos profissionais com os princípios e diretrizes do SUS (Brasil, 2006). 
A nova maneira de interpretar a educação dos profissionais da saúde explicitou a inadiável necessidade de redefinição do ambiente onde essa educação deve acontecer. Não se concebem mais processos educativos cujo único ambiente é o hospital universitário, pois se compreende que, por maior 'segurança' que esse ambiente possa oferecer aos profissionais, é necessário romper com a dissociação entre clínica e política, o que é possível apenas em ambientes e situações reais (Ceccim e Ferla, 2008). A educação dos profissionais da saúde pode e deve acontecer, portanto, em um ambiente privilegiado no que se refere à capacidade de realização das vivências essenciais ao aprendizado, o próprio SUS.

Uma compreensão melhor e mais profunda dos processos educativos apresenta-se como fundamental no cenário atual da saúde no Brasil. Ao se concluir que as práticas educativas estavam desarticuladas das necessidades dos usuários, o desafio é criar, recriar, propor e modernizar tecnologias educacionais capazes de atender a tais necessidades.

A dimensão da complexidade de formar adequadamente os profissionais do sistema público de saúde pode ser percebida por meio de uma leitura, ainda que superficial, do contexto nacional contemporâneo. Questões como urbanização e o aumento da densidade em polos urbanos, envelhecimento da população, degradação ambiental e perda da biodiversidade são apenas alguns dos importantes fatores que influenciam a saúde da população. Decorrem dessa realidade situações envolvendo questões como a saúde da mulher, do idoso, obesidade, hipertensão, dentre tantas outras que se apresentam diariamente aos profissionais de saúde e exigem destes uma atitude resolutiva.

É fato que ampliar a compreensão sobre a complexidade e a importância da educação/formação dos profissionais da saúde exige uma aproximação das vivências ocorridas dentro dos serviços de saúde. Este texto originou-se, pois (e nisto também se constitui), num momento de aproximação dessas vivências. O cenário é o próprio SUS e os atores são todos aqueles que o compõem. Partimos da compreensão de que não basta saber o que fazer, isto é, educar/formar os profissionais com base nas necessidades dos usuários do serviço. Como questiona Nóvoa:

Como fazer aquilo que dizemos que é preciso fazer? O que será necessário fazer para dar coerência aos nossos propósitos, materializando na prática o consenso que vem se elaborando em torno da aprendizagem docente e do desenvolvimento profissional? (Nóvoa, 2009, p. 16)

Precisamos investir esforços na compreensão sobre como essa educação tem acontecido, que práticas pedagógicas se estabelecem e quais concepções pedagógicas têm norteado essas práticas. É a começar dessa compreensão que 
será possível contribuir com a oferta de tecnologias educacionais dinâmicas, assim como o são as demandas sociais do setor.

Objetivamos, pois, compreender as práticas educativas dos preceptores em saúde, justamente pelo fato de que são esses os profissionais que atuam como formadores/educadores dos estudantes, futuros profissionais do serviço público de saúde. A compreensão pretendida aqui é a que se pode ter pelo acesso às práticas educativas de profissionais/preceptores, a qual será mediada pelo pensamento de importantes autores das áreas da Saúde Coletiva, Educação e Educação em Saúde (Alcindo Ferla, Antonio Nóvoa e Ricardo Ceccim), áreas essas que, ao se imbricarem e se permearem, criam o cenário ideal para a reflexão sobre os processos educativos em saúde.

\section{Olhar, ver e ouvir o cotidiano do trabalho: uma interação necessária na formação em saúde}

O papel dos preceptores em saúde na relação cotidiana com os alunos e com os usuários da rede de serviços, longe de se revestir de um significado meramente técnico (assistencial), se inscreve numa prática de múltiplas dimensões, o que legitima a abordagem metodológica qualitativa. O estudo 3 aconteceu na unidade básica de saúde de Nazaré, que pertence ao Distrito de Saúde Oeste, no município de Natal, no Rio Grande do Norte. A disciplina Saúde e Cidadania, a qual é ofertada a todos os cursos de graduação da área da saúde na Universidade Federal do Rio Grande do Norte (UFRN) serviu como um 'meio' para a compreensão dos processos educativos que se realizam durante a formação dos preceptores e dos estudantes da área da saúde.

Considerando nosso interesse em alcançar, em todas as dimensões possíveis, o objeto em estudo, isto é, os processos educativos realizados pelos preceptores junto aos estudantes de graduação da área da saúde, escolhemos, entre as diferentes técnicas disponíveis para a abordagem qualitativa, a entrevista semiestruturada e a observação direta, por entendermos que elas nos permitiriam conduzir as perguntas e complementá-las, quando necessário, possibilitando aos entrevistados melhor compreensão das questões abordadas (Ludke e André, 1986, p. 34).

Optamos ainda pela construção e organização de instrumentos específicos para a condução da pesquisa, os quais contemplaram aspectos próprios da formação no campo da saúde. As entrevistas, gravadas em equipamento de áudio, foram realizadas com dois preceptores de cada uma das três unidades de saúde elencadas. Uma caracterização resumida desses profissionais mostrou que os seis preceptores entrevistados têm formação em Medicina, Enfermagem e Odontologia e têm idade acima de quarenta anos; 
demonstram grande conhecimento sobre as condições sociais, econômicas e sanitárias do bairro onde atuam.

O exame detalhado do material das entrevistas foi realizado com base na análise hermenêutica-dialética, proposta por Habermas, a partir de um diálogo com Gadamer (1987). De acordo com Minayo (2010), a hermenêutica-dialética representa uma possibilidade de alcançar as dimensões e a dinâmica das relações que têm por objeto a saúde.

De acordo com Ferreira (1998), a hermenêutica significava, originalmente, teoria ou método de interpretação da Bíblia e de outros textos religiosos. Friedrich Schleiermacher (1768-1834) formulou uma teoria da interpretação dos textos e do discurso, que Wilhelm Dilthey (1833-1911) aplicou a todos os atos e produtos humanos e Hedegger estendeu ao ser humano. A hermenêutica está, portanto, associada à ideia de círculo hermenêutico: não podemos compreender completamente um todo (por exemplo, um texto filosófico), a menos que entendamos as suas partes, ou completamente as partes, a menos que entendamos o todo. Heidegger e Hans-Georg Gadamer (1900-2002) fizeram disso uma característica de todo o conhecimento e atividades humanos.

A hermenêutica representa, para Minayo (2010), a arte da compreensão. A autora, tomando como base os estudos de Gadamer (1999), afirma que, de acordo com a abordagem hermenêutica, "as ideias de alteridade, entendimento e a noção de mal-entendido são possibilidades universais tanto no campo científico como no mundo da vida" (Minayo, 2010, p. 329).

A dialética, por sua vez, é apresentada por Minayo (2010) como a arte do estranhamento e da crítica. Ao referir-se ao percurso histórico da dialética, bem como ao velho dilema entre quantitativo e qualitativo, a autora afirma que a oposição entre quantidade e qualidade é dialética e complementar.

A articulação entre as abordagens hermenêutica e dialética representa, pois, uma possibilidade de reflexão fundamentada na práxis, constituindo-se numa importante técnica para a compreensão e análise em pesquisas qualitativas, capaz de atribuir compreensão e crítica aos estudos da realidade social (Minayo, 2010, p. 343).

A análise hermenêutica-dialética possibilitou, pois, compreender como os vários elementos presentes no contexto contribuem para configurar as práticas educativas/formativas dos preceptores em saúde, bem como as tecnologias educacionais que aí se utilizam. A dimensão da compreensão que aqui se estabeleceu pode ser apresentada por meio da citação a seguir:

(...) compreender implica a possibilidade de interpretar, de estabelecer relações e extrair conclusões em todas as direções. Mas compreender acaba sendo sempre compreender-se (Minayo, 2010, p. 337). 
A observação direta, realizada durante oito encontros de quatro horas, totalizando 32 horas, visou acompanhar, in loco, as vivências educativas ocorridas nas unidades de saúde. Após o primeiro encontro com o grupo de preceptores e estudantes, sentimos necessidade de maior sistematização dessa técnica, considerando o que é apresentado por Ludke e André (1986, p. 25):

Para que se torne um instrumento válido e fidedigno de investigação científica, a observação precisa ser antes de tudo controlada e sistemática.

Assim, após a delimitação do nosso objeto, procuramos definir o foco de nossa observação, que é a formação educativa ocorrida no âmbito da atividade de preceptoria em saúde, em interação com os estudantes e a comunidade, sendo esta um ambiente privilegiado da integração ensino-serviço.

Inicialmente, os atores envolvidos nas atividades observadas foram informados sobre os objetivos da pesquisa, como também sobre as especificidades da observação direta. Essa estratégia possibilitou a compreensão de aspectos do cotidiano que não se revelaram nos depoimentos dos preceptores durante as entrevistas, permitindo maior aproximação da "perspectiva dos sujeitos" (Ludke e André,1986, p. 26). Houve, portanto, uma inserção no ambiente estudado, a qual gerou registros construídos a partir do olhar, do ver e do ouvir o cotidiano do processo de formação dos estudantes sob a orientação dos preceptores.

Este estudo está vinculado à pesquisa de mestrado intitulada "Integração ensino e serviço: dizer e fazer saúde na Atenção Básica", registrada e aprovada pelo Comitê de Ética do Hospital Universitário Onofre Lopes, de acordo com o certificado número 14196913.2.0000.5292.

\section{Preceptoria em saúde: a educação que se faz no interior do serviço público em interação com a comunidade}

A educação dos profissionais da saúde é um assunto de elevada importância quando se concebe um serviço público de saúde que contemple as necessidades dos usuários em sua complexidade. A PNEPS ampliou, a partir de 2003, o debate sobre o tema e, ao determinar a priorização da educação da saúde como uma ação finalística, rompeu com a ideia de 'capacitação de recursos humanos', até então adotada pelas instituições formadoras. A formação dos profissionais da saúde, tradicionalmente exercida mais por referências externas do que por referências internas ao trabalho na saúde, inverteu essa lógica, possibilitando instituir práticas educativas sensíveis às necessidades educativas. 
Um estudo realizado por Ceccim (2005a, p. 976) afirma que a produção da PNEPS "representou o esforço de cumprir uma das maiores metas formuladas pela saúde coletiva no Brasil: tornar a rede pública de saúde uma rede de ensino-aprendizagem no exercício do trabalho". Em estudo posterior, Ceccim e Ferla (2009, p. 454) afirmam que "programas de formação plenos de conteúdos e que possuem ritos a serem percorridos e vencidos em condições e ritmos de tempos preestabelecidos" não contribuirão para a conquista das mudanças necessárias à construção de um trabalhador coletivo.

Alcançar o status almejado pela PNEPS significa investir em permanente compreensão sobre os processos pedagógicos que se desenvolvem no interior das instituições formadoras dos profissionais da saúde. O desafio é agir crítica, reflexiva e propositivamente, procurando desenvolver ações formativas que partam da realidade que se apresenta nos cenários sociais brasileiros, tendo como pressuposto que o Sistema Único de Saúde (SUS) é o espaço privilegiado dessa formação.

Antonio Nóvoa, em seu estudo sobre a formação de professores da área da educação, citou como exemplo a ser seguido pelos educadores o modo de formação dos médicos. O autor afirmou: “O modo como a sua preparação está concebida nas fases da formação inicial, de indução e de formação em serviço talvez nos possa servir de inspiração" (Nóvoa, 2009, p. 17).

É interessante perceber que a área da saúde pode representar uma referência para outras profissões, como a dos educadores, por exemplo. Entretanto, se, por um lado, isso pode significar motivo de 'orgulho' para os profissionais da saúde, talvez seja possível, por outro lado, num movimento de alteridade, perguntar: como os conhecimentos da pedagogia podem contribuir (ou têm contribuído) com a formação dos profissionais da saúde?

Não seria propriamente uma novidade afirmar que a pedagogia já contribui com a formação dos profissionais da saúde. É possível que a novidade esteja em identificar essas práticas pedagógicas, revelar o contexto e o ambiente em que acontecem, atribuir-lhes a real importância e eficácia e, principalmente, apresentar a reflexão necessária para transformá-las em uma possível "Pedagogia da Educação em Saúde".

A intercessão entre as áreas da educação e da saúde tem sido interpretada por alguns estudiosos (Ceccim, 2005; Trajman et al., 2009) como estratégica para a proposição de tecnologias inovadoras de formação profissional no campo da saúde. Entretanto, os processos de formação dos educadores da saúde ainda se mostram deficientes. A composição dos ambientes de aprendizagem apresenta-se inadequada, prevalecendo, ainda, as maneiras e os valores tradicionais que produzem um bloqueio sobre os processos criativos, os quais se pretendiam dinâmicos e cuja orientação deveria acontecer a partir da complexidade do setor. 
Ceccim e Ferla (2008, p. 449), ao apresentarem a imagem de uma 'mandala' como alternativa à tradicional e estática pirâmide assistencial usada para representar a orientação dos profissionais da saúde, afirmam:

Uma reorientação da educação dos profissionais da saúde deve ocorrer e passar pela reversão da matriz vigente de pensamentos e de práticas, desenvolvendo dispositivos também dinâmicos e extremamente flexíveis para escutar, retraduzir e trabalhar essas necessidades, tendo em vista a construção de uma cadeia de cuidados progressivos à saúde para os usuários, visando à garantia de acesso a todas as tecnologias disponíveis para enfrentar as doenças e prolongar a vida (Ceccim e Ferla, 2008, p. 449).

Analisando a possibilidade de, com base no pensamento desses autores, identificar um caminho, uma ponta desse emaranhado novelo da formação em saúde que nos possa conduzir a uma reflexão sobre as possibilidades dessa formação é que 'pinçamos', de uma extensa lista de atores, a figura do preceptor em saúde. É a partir do trabalho dos profissionais comprometidos com a atividade de preceptoria em saúde que pretendemos caminhar nas trilhas que possam nos conduzir para ampliar um pouco mais o conhecimento sobre essa possível 'pedagogia da educação em saúde'.

O reconhecimento legal da função do preceptor em saúde - atividade que tem como principal atribuição "ensinar a clinicar, através de instruções formais e com objetivos e metas claramente definidos" (Botti e Rego, 2008) pode significar, neste contexto, uma via de aprofundamento do compromisso com o SUS por parte das instituições formadoras, entendendo-se que estas não podem se isentar da missão de oferecer uma educação de qualidade, isto é, uma educação capaz de responder às crescentes demandas sociais do setor saúde.

De acordo com Trajman et al. (2009), o grande desafio dos preceptores está situado junto à Atenção Primária em Saúde e consiste em proporcionar aprimoramento aos estudantes, oferecendo apoio na busca por qualificação profissional que deverá resultar numa melhor assistência ao usuário, sua família e à comunidade. Esse processo demanda uma formação/aculturação pedagógica para além das funções técnicas atribuídas aos preceptores.

A principal preocupação do preceptor é com os aspectos do ensino-aprendizagem do profissional da saúde, favorecendo a aquisição de habilidades e competências pelos estudantes, em situações clínicas reais, no próprio ambiente de trabalho (Botti e Rego, 2008). A atuação e a formação desse profissional necessitam ser priorizadas no que se refere à compreensão sobre sua capacidade de influenciar e produzir novas práticas no ensino da saúde. 


\section{Integração ensino-serviço: criando novas possibilidades de atuação docente na saúde}

A abordagem aqui apresentada contemplou, na maioria das vezes, a díade preceptor-educando (ou educador-educando, entendendo o preceptor em sua função de educador). Isso não significa, entretanto, que a educação da saúde se dá numa relação biunívoca. Ao contrário, os processos educativos que se estabelecem no interior do serviço público de saúde são amplos, envolvem atores de diferentes setores e, portanto, devem ser considerados em toda a sua complexidade e singularidades.

Os preceptores em saúde veem a si mesmos como os profissionais mais próximos dos alunos e, por isso, mais capacitados para fazer a ponte teoria-prática no processo ensino-aprendizagem. A atividade da preceptoria se dá, portanto, dentro dos processos de trabalho estabelecidos para os profissionais do serviço público, significando a oportunidade de colocar o estudante em contato com a realidade local.

Ao acompanhar os estudantes em seu processo formativo-educativo, os preceptores oportunizam a estes - e a si mesmos - conhecer o lócus de atuação do profissional da saúde pública: a comunidade, seus problemas sociais, ambientais, sanitários; as relações que se estabelecem entre profissional e paciente; os anseios da população em relação à saúde; as diferentes concepções sobre temas como educação, saúde, cidadania; os equipamentos sociais e sua forma de utilização pela comunidade. De maneira dirigida - ou não -, preceptores e estudantes estabelecem um debate a respeito desses temas centrais, passando de uma visão de profissionais e estudantes para a visão de aprendentes do 'fazer saúde' para as coletividades.

Os relatos dos preceptores em saúde evidenciaram mudanças significativas na atividade profissional a partir do início de sua atuação junto aos estudantes das diferentes áreas. O sentido de responsabilidade como um valor da profissão foi um ponto de destaque nas entrevistas realizadas. Estabeleceu-se, para os preceptores, a necessidade de buscar novos conhecimentos, os quais deveriam extrapolar sua área específica de formação acadêmica e interagir com as demais áreas. Com a convicção de que o aprendizado é permanente e incessante, os profissionais buscam identificar pontos de interesse dos alunos, objetivando tornar o aprendizado da saúde mais interessante, prazeroso e significativo.

A apreensão do conhecimento deve acontecer de maneira ampla, não podendo fundamentar-se no saber unilateral, mas em interação com o outro. A qualidade do que se ensina/aprende está diretamente ligada à capacidade de interação entre os atores. Para os preceptores em saúde, é no permitir ao outro acessar seu espaço de trabalho, suas ideias e suas experiências que reside a possibilidade de ampliação, melhorias e sucesso no trabalho da saúde. 
Compreendendo que formar profissionais para atuar na saúde pública é uma tarefa complexa e, portanto, de múltiplas faces, e com a intenção de possibilitar práticas educativas inovadoras, capazes de promover motivação e o despertar para a capacidade criativa dos preceptores e alunos, seria coerente refletir sobre quais conteúdos ensinar/aprender e quais estratégias usar.

A necessidade de garantir uma formação ampla para os profissionais da saúde nos leva a compreender que o trabalho em equipe se constitui em um conteúdo e, como tal, deve ser ensinado por meio de uma estratégia pedagógica vivenciada no próprio trabalho. A ideia é garantir a compreensão de que não é possível fazer saúde de maneira individualizada, embora se identifiquem resistências entre alguns profissionais cujo processo histórico mostra a supervalorização de algumas profissões em detrimento de outras.

Ao responder à pergunta “O que mudou na sua prática profissional após assumir a atividade de preceptoria?", uma odontóloga da Estratégia Saúde da Família, que atua como preceptora de alunos dos cursos de Enfermagem, Nutrição, Farmácia, Odontologia e Medicina, respondeu: “Nós, profissionais odontólogos, fomos formados para trabalhar com a boca do paciente; já neste trabalho com os alunos a gente aprende a ver a pessoa como um todo".

Essa verbalização revela que a formação na perspectiva do trabalho em equipe e o envolvimento multiprofissional no ensino da saúde pode significar mudança não apenas na formação dos estudantes, mas, principalmente, a construção de uma nova visão dos profissionais formados numa educação tradicional, conteudista e fragmentada. O trabalho multiprofissional que se realiza nas unidades de saúde da família tem se mostrado não apenas mais eficaz para proporcionar maior acesso aos conteúdos e saberes necessários à prática do trabalho na saúde, mas, também, infinitamente mais prazeroso. Em diferentes momentos surgiram, durante as entrevistas com os preceptores, colocações como: "eu despertei para a importância do trabalho em equipe"; "quero contribuir para a construção do conhecimento de outras pessoas". Uma interpretação pedagógica dessas verbalizações revela o prazer que os profissionais têm de poder interagir entre si, com os alunos e a comunidade, numa relação multiprofissional que possibilita fazer mais do que se espera, extrapolar os limites, atingir o alvo.

Os registros da observação direta também revelam importantes aspectos da formação em saúde. Sua interpretação partiu sempre da compreensão dos possíveis avanços para a superação de práticas pedagógicas arcaicas que, ao invés de produzirem motivação, se traduzem em momentos de desinteresse para os estudantes. A observação revelou desde docentes que atuam de maneira que deixa clara sua preferência por modos de ensinar, pautados numa pedagogia tradicional, privilegiando a histórica maneira de formar e formar-se professor dentro da concepção tradicional da 'transmissão de 
conhecimentos', até situações que revelaram ruptura com os valores da educação tradicional, pela adoção de metodologias inovadoras por parte de alguns preceptores.

De um modo geral, observou-se que existe o grande desafio de transformar os interesses dos alunos em aprendizado real; é necessário lançar mão de recursos capazes de dinamizar as atividades, os quais devem ser escolhidos de acordo com o assunto debatido. É preciso refletir sobre como transformar as situações pedagógicas em 'oportunidades de inovação no ensino da saúde'. É fundamental que os docentes sejam capazes de inovar, dinamizar, problematizar as situações de maneira a transformá-las em 'momentos de rico aprendizado', e que eles não sejam limitados pelo currículo disciplinar nem acabem por atuar de maneira a promover pouca inovação; é necessário enfrentar o 'medo' de experimentar e ousar, para que sejam capazes de extrapolar os limites da educação pautada nos valores tradicionais, acessando novas ideias, maneiras diversificadas de agir para superar o modo de educar pautado na perspectiva de que 'um é o que ensina, outro é o que aprende'.

O desafio que se estabeleceu na prática desses profissionais foi o de desapegar-se das velhas e tradicionais formas de ensinar/aprender; é necessário assimilar e internalizar novas maneiras de formar e formar-se. Tomando como ponto de apoio o que é afirmado por Nóvoa (2009, p. 13), a aprendizagem e o desenvolvimento profissional docente são assegurados por uma articulação da formação inicial e da formação em serviço, numa perspectiva de aprendizagem ao longo da vida.

O trabalho da preceptoria em saúde tem-se revelado dinâmico e com imensa potencialidade para uma educação/formação adequada às necessidades já amplamente descritas aqui. A observação do espaço das salas de aula mostrou ambientes organizados de maneira compatível com uma pedagogia progressista. O uso de mapas, cartazes, descritores; a organização dos espaços onde as atividades se desenvolvem revelou uma visão crítica dos preceptores, os quais valorizam o trabalho coletivo, multiprofissional e refletem sobre o fazer pedagógico no momento exato desse fazer, mostrando que teoria e prática não são processos isolados.

Os registros feitos mediante observação revelaram, em outro momento, uma postura docente aberta a captar o outro em sua dimensão humana, emocional, de saberes prévios adquiridos ao longo da vida, de suas experiências e inquietações, ratificando o conceito de educação permanente como sendo, essencialmente, aprendizagem no trabalho.

As teorias da educação em saúde atualizam-se - não no sentido de serem confirmadas, mas no de sua conexão com as práticas - a partir das indagações, críticas e reflexões que surgem no debate entre os atores que ensinam e aprendem juntos. A teoria não alcança, por mais abrangente que possa 
parecer, a complexidade da formação. Isso é facilmente compreendido quando observamos o volume de explicações produzidas e a certeza de que nenhuma delas contém a verdade absoluta, mas apenas pontos de vista.

A educação em saúde é um processo e, como tal, está em permanente transformação. A complexidade de seu objeto, bem como o fato de se desenvolverem em seu interior processos conflitantes, nos diz que ainda há muito a aprender sobre os movimentos que constituem esse espaço de ensino-aprendizagem. O caminho que se faz na direção desse conhecimento pode, por mais estranho que possa parecer, revelar conhecimento, não numa perspectiva de linearidade, mas como resultante das vivências de todos os envolvidos e à revelia do que estava estabelecido como correto, aceitável ou esperado.

A formalização da atividade de preceptoria imprimiu um novo significado ao trabalho desses profissionais. Os desafios que se lhes apresentaram trouxeram novas possibilidades de autoformação, algo até então não identificado por eles. Os profissionais passaram a atuar a partir da perspectiva da interação com o outro, sendo este outro o colega profissional de uma área diferente da sua; ou o usuário do sistema; ou a família desse usuário.

A reflexão de Nóvoa (2009, p. 20) a respeito da formação dos professores e o sentimento de pertencimento e de identidade profissional reforçado a partir dos movimentos pedagógicos e das comunidades de práticas mostra que esse mesmo sentimento pode ser identificado no dia a dia do trabalho da preceptoria em saúde. As metodologias adotadas possibilitam a esses profissionais apropriar-se dos processos de trabalho, transformando-os em práticas concretas de intervenção e produzindo a reflexão coletiva, que, por sua vez, produz o sentido de desenvolvimento profissional almejado pela PNEPS, que é o de ensino-aprendizagem no exercício do trabalho.

As vivências que se realizam no interior dos serviços de saúde com base na atuação da preceptoria representam, em última análise, uma prática bastante reclamada pelos estudiosos da Educação em Saúde, que é a integração entre os serviços de saúde e a academia. As dificuldades nessa integração, como debatido por Trajman et al. (2009), foram identificadas no depoimento de um dos preceptores em saúde:

(...) a qualificação profissional, com o apoio da gestão e das instituições parceiras, através da universidade, cada dia capacitando, através dos mestrados profissionais, para que a gente possa melhorar a qualidade da assistência prestada no serviço. A gente fica triste, porque, assim, se não tiver o apoio do gestor, principalmente local, se não tiver a compreensão dos próprios profissionais, você se desgasta enquanto profissional, para fazer uma coisa que é nosso papel. A gente 
não está fazendo aqui para ser bonito, ser destaque. É nosso papel, a educação permanente, a educação continuada (P-1).

A relação dos preceptores com o trabalho que realizam na formação dos estudantes não se resume à mera transmissão de conhecimentos anteriormente adquiridos. Preceptoria está relacionada com autoformação e há o interesse de integração com a universidade como uma maneira de interagir com a pós-graduação e entrar em contato com a pesquisa, como se nota no depoimento de um preceptor:

Ser preceptor em saúde é participar da formação dos alunos e contribuir com a formação dos alunos da universidade, e também uma oportunidade para a gente, como preceptor, estar sempre se atualizando, estar entrando em contato com as coisas da universidade, da academia, né, que a gente não fica tão distante da academia (P-5).

A integração entre o ensino e os serviços de saúde se estabelece, portanto, numa relação de necessidades - e possibilidades - identificadas por ambos os lados. Se, de um lado, os alunos se beneficiam com o conhecimento compartilhado pelos preceptores, estes, por sua vez, esperam usufruir das oportunidades de ampliação da formação, mediante a inserção em todas as modalidades formativas que a academia possa oferecer.

\section{Conclusão}

A noção que os preceptores têm sobre a importância do próprio trabalho como profissionais que atuam diretamente no processo de formação/educação dos estudantes da área da saúde está baseada na reflexão que fazem sobre o processo educativo vivenciado no SUS. Grande parte desses profissionais formou-se nas décadas de 1980 e 1990 e compara aquele momento com as facilidades apresentadas pelas diferentes tecnologias do mundo atual. Para eles, um importante desafio para conduzir adequadamente a formação dos estudantes encontra-se na capacidade de mobilizá-los para saírem de trás dos muros onde se encontram e, extrapolando seus limites, estabelecerem uma relação real com a comunidade.

Identifica-se um esforço no sentido de assimilação da PNEPS por parte dos profissionais que atuam como formadores dos estudantes da área da saúde. É possível que a busca pelo conhecimento de novas metodologias de ensino, associada ao aprofundamento dos conhecimentos da área da pedagogia e à prática do ensino multiprofissional signifiquem um avanço no 
campo da Educação em Saúde. Não seria redundante afirmar que mobilizar os estudantes para o trabalho em equipe ainda representa um desafio, considerando as ultrapassadas e tradicionais metodologias educacionais que formavam para a competição e o sucesso individual, as quais ainda estão fortemente presentes no discurso e nas ações dos educadores.

Além de todos esses desafios, existe a necessidade de maior apropriação do SUS, a qual pode se dar com a ampliação e o estreitamento das relações entre a academia e os serviços de saúde.

O campo da Educação em Saúde tem-se revelado, portanto, um espaço de constante quebra do paradigma das certezas, das verdades absolutas, um espaço gerador da emergência de um novo olhar sobre a complexa necessidade de formar profissionais capazes de lidar com a também complexa lista de desafios da saúde pública no Brasil.

Resumen La discusión actual sobre educación y salud viene mostrando la necesidad de mayor integración entre los servicios de salud y la academia. La Política Nacional de Educación Permanente en Salud propone la diseminación de capacidad pedagógica en el Sistema Único de Salud, de modo que la red pública de salud pase a constituir un espacio de enseñanza-aprendizaje en el ejercicio del trabajo. El objetivo que se estableció fue el de conocer el proceso de integración entre la enseñanza y el servicio de salud en la Atención Básica en Salud, a partir de la actuación de los preceptores en el proceso de formación de los estudiantes de los cursos de graduación del área de la salud en la Universidad Federal de Río Grande do Norte. La entrevista semiestructurada y la observación directa fueron las técnicas seleccionadas, y el análisis de los datos, realizado en base al abordaje hermenéutico y dialéctico, mostró que la actuación de los preceptores constituye una importante estrategia para permitir la integración enseñanza-servicio. La conclusión señala hacia la comprensión de que los profesionales involucrados en la preceptoría se educan mientras educan, así como el proceso educativo está permeado por saberes y experiencias heterogéneas, factor altamente favorable a la formación de los estudiantes y profesionales.

Palabras clave educación; salud; política nacional de educación permanente en salud; preceptoría. 


\section{Notas}

1 Fundação Oswaldo Cruz, Instituto Leônidas e Maria Deane, Manaus, Amazônia, Brasil. <aniziaaneta@gmail.com>

Correspondência: Rua Teresina, 476, Adrianópolis, CEP 69057-070, Manaus, Amazônia, Brasil.

2 Universidade Federal do Rio Grande do Norte, Centro de Ciências da Saúde, Departamento de Odontologia.

<socorrocfa@hotmail.com>

${ }^{3}$ Este artigo é um resultado parcial da pesquisa de mestrado intitulada, inicialmente, A formação educativa dos preceptores em saúde: um estudo na rede básica de saúde no município de Natal-RN. A referida pesquisa foi concluída e a dissertação de mestrado foi defendida em fevereiro de 2014. O título definitivo da dissertação passou a ser, por solicitação da banca examinadora, Integração ensino e serviço: dizer e fazer saúde na atenção básica.

\section{Referências}

BOTTI, Sérgio H. O.; REGO, Sérgio. Preceptor, supervisor, tutor e mentor: quais são seus papéis? Revista Brasileira de Educação Médica, Rio de Janeiro, v. 32, n. 3, p. 363-373, 2008.

BRASIL. Ministério da Saúde. Política Nacional de Educação Permanente em Saúde. Brasília: Ministério da Saúde, 2009. 64 p. (Série B. Textos Básicos de Saúde). (Série Pactos pela Saúde 2006, v. 9).

CECCIM, Ricardo B. Descentralização e disseminação de capacidade pedagógica na saúde. Ciência \& Saúde Coletiva, Rio de Janeiro, v. 10, n. 4, p. 975-986, out.-dez. 2005.

CECCIM, Ricardo B.; FERLA, Alcindo A. Educação e saúde: ensino e cidadania como travessia de fronteiras. Trabalho, Educação e Saúde, Rio de Janeiro, v. 6, n. 3, p. 443-456, 2008.

FERREIRA, Aurélio B. H. Dicionário Aurélio básico da língua portuguesa. Rio de Janeiro: Nova Fronteira, 1988.
LUDKE, Menga.; ANDRÉ, Marli E. D. A. Pesquisa em educação: abordagens qualitativas. São Paulo: EPU, 1986.

MINAYO, Maria C. S. O desafio do conhecimento: pesquisa qualitativa em saúde. 12. ed. São Paulo: Hucitec, 2010.

NÓVOA, Antonio. Professores: imagens do futuro presente. Lisboa: Educa Editora, 2009.

TRAJMAN, Anete et al. A preceptoria na rede básica da Secretaria Municipal de Saúde do Rio de Janeiro: opinião dos profissionais de saúde. Revista Brasileira de Educação Médica, Rio de Janeiro, v. 33. n. 1, p. 24-32, 2009.

Recebido em 16/08/2013

Aprovado em 24/11/2014 